Research Article

\title{
Anatomically and Dielectrically Realistic 2.5D 5-Layer Reconfigurable Head Phantom for Testing Microwave Stroke Detection and Classification
}

\author{
Tomas Pokorny ${ }^{D},{ }^{1}$ David Vrba $\left(\mathbb{D},{ }^{1}\right.$ Jan Tesarik ${ }^{D},{ }^{1}$ Dario B. Rodrigues ${ }^{(D},{ }^{2}$ and Jan Vrba ${ }^{1}$ \\ ${ }^{1}$ Department of Biomedical Technology, Faculty of Biomedical Engineering, Czech Technical University in Prague, Prague, \\ Czech Republic \\ ${ }^{2}$ Department of Radiation Oncology, University of Maryland School of Medicine, Baltimore, MD, USA
}

Correspondence should be addressed to Tomas Pokorny; tomas.pokorny@fbmi.cvut.cz

Received 19 October 2018; Accepted 17 July 2019; Published 25 August 2019

Guest Editor: Alvaro Rocha

Copyright (c) 2019 Tomas Pokorny et al. This is an open access article distributed under the Creative Commons Attribution License, which permits unrestricted use, distribution, and reproduction in any medium, provided the original work is properly cited.

\begin{abstract}
This work presents the design and manufacturing of an anatomically and dielectrically realistic layered phantom of the human head that allows the insertion of ischemic and hemorrhagic stroke phantom models. A 2.5D physical phantom was designed using a representative anatomical image of the human head, which was simplified into 5 different layers that mimic the scalp, skull, cerebrospinal fluid, brain, and stroke regions in terms of anatomy and dielectric properties. Apart from the brain phantom, all other layers consist of a mixture of polyurethane rubber, graphite powder, and carbon black powder. The brain phantom is in the liquid form to facilitate the insertion of different stroke models (ischemic or hemorrhagic) with different positions and shapes. Phantoms were designed with dielectric properties valid within the frequency range $0.5-3.0 \mathrm{GHz}$, which is relevant for microwave stroke detection and classification. Molds for casting individual parts of the phantom were printed in 3D. The presented phantom is suitable for the development and testing of microwave systems and algorithms used in the detection and classification of vascular events relevant to stroke diagnosis.
\end{abstract}

\section{Introduction}

A stroke can cause lasting brain damage, long-term disability, and even death [1]. Recent developments in microwave imaging (MWI) have shown the potential for MWI to be used as a tool for detection and classification of different stroke types [2]. This technology is typically compact, light, portable, noninvasive, and inexpensive, which are advantages compared with traditional imaging techniques such as computed tomography (CT) or magnetic resonance (MR) $[3,4]$. However, testing this imaging technique in patients who have their life at risk is not possible. The only solution is to develop an anatomically and dielectrically realistic human head phantom with layers that mimic the anatomy and dielectric properties of human head tissues as close as possible.
Dielectric properties vary depending on the stroke type, which can be classified into two main categories: hemorrhagic and ischemic. Ischemic strokes are caused by the interruption of blood flow within a region of the brain and are more common than hemorrhagic strokes, which are triggered by blood leakage/bleeding phenomena [1]. The dielectric properties of the blood are higher than the ones of the white and gray matter [5]. Furthermore, acute ischemic stroke causes a decrease in the dielectric properties of the brain tissue and the degree of such changes depends on the stage of ischemic disease [6]. Microwave systems should then be able to not only detect strokes but also classify different stroke types due to their different dielectric properties [2].

Most head phantoms [7-9] are made of water-based substances that consist of a single layer with dielectric 
parameters corresponding to the average of human head tissues, which vary widely. Anatomical phantoms that contain individual tissues are available [10-12], but these phantoms are significantly simplified and use plastic materials, gels, and water-based substances that are not timestable [13]. Furthermore, the insertion of stroke phantoms in different positions and their reuse are not possible with such phantoms.

Improved phantom properties can be obtained by using silicone or polyurethane rubber in combination with conductive powders [14-16]. These phantom materials show long-term stability both in shape and dielectric properties. They are also suitable for creating complex shapes and thin layers, but the ones available are still composed of one layer with average dielectric properties of tissues in the brain. In reference [17], a dielectrically and anatomically realistic solid 3D phantom was fabricated, resulting in a time-stable phantom that accommodates the insertion of stroke phantoms. However, only two predefined positions for the stroke phantom can be used and the head model only has two layers of tissue: an outer homogeneous layer exhibiting average dielectric properties of tissues outside the brain, that is, skin, fat, skull cortical bone, skull cancellous bone, meninges, and cerebrospinal fluid (CSF), and an inner homogeneous layer exhibiting average dielectric properties of the gray matter, white matter, and CSF.

Clearly, a more detailed and versatile human head phantom is required for testing microwave systems. The human head has several tissue layers: epidermis, scalp (skin, connective tissue, galea aponeurotic layer, loose areolar connective tissue layer, and pericranium), skull (outer middle and inner table layers), meninges (dura mater, arachnoid mater, and pia mater), and cerebrospinal fluid and brain parenchyma (white and gray matter) [18]. In this work, we propose a 5-tissue layer with scalp, skull, CSF, brain, and stroke compartments, where the stroke phantom can be positioned anywhere in the brain as is the case of naturally occurring strokes.

\section{Materials and Methods}

2.1. Phantom Mold Design. To fabricate a multilayer head phantom, we designed concentric phantom molds with anatomically realistic shapes. To this end, we analyzed MR scans from The Population Head Model V1.0 of the IT'IS Foundation database $[19,20]$ and selected model number 122317 due to its average head size. Using this model, we defined the baseline image for the molds production as the axial cross section that provided the largest brain portion of the head model (Figure 1(a)). The different layers of the human head were then segmented from the baseline image using the software 3D Builder (Microsoft Corporation) as shown in Figure 1(b). The molds are intended to be printed in $3 \mathrm{D}$. Thus, the anatomy had to be simplified to create layers that are both feasible to be printed and mechanically stable. From our experience, a minimum thickness of $2 \mathrm{~mm}$ for each layer was chosen. The resulting simplified geometry can be seen in Figure 1(c) with 5 tissue layers: scalp, skull, CSF, brain, and a stroke model added a posteriori.
The $2.5 \mathrm{D}$ rendering of the phantom molds is shown in Figure 2 with a height of $20 \mathrm{~cm}$. The outer shell (Figure 2(a)) dictates the head shape, and the molds in Figures 2(b), 2(c), and 2(d) will shape the skull, CSF, and brain regions, respectively. The mold for the stroke phantom (Figure 2(e)) is represented by a cylinder with a cross-sectional diameter of $4 \mathrm{~cm}$. All phantoms will be made in the solid form, except the brain region which will be in the liquid form. A liquid brain region will allow immersion of stroke phantoms of different sizes and shapes.

\subsection{Definition of Dielectrically Equivalent Phantom Models.} The solid phantoms were prepared using mixtures of polyurethane rubber, graphite powder, carbon black powder, and acetone (Table 1). These materials were chosen due to their mechanical stability and their ability to match the dielectric properties of human tissues [21]. In total, 28 mixtures were fabricated in different proportions (Table 1).

A total of 28 mixtures were produced in 6 series in different compositions listed in Table 2. In each series, the weight percent of one component (graphite powder, carbon black powder, or acetone) was increased while the weight percent of the other component (graphite powder, carbon black powder, and acetone) was kept constant.

Dielectric properties of mixtures were measured using the SPEAG Dielectric Assessment kit (DAK, Schmid \& Partner Engineering AG, Switzerland) with the probe DAK-12 in the frequency range $0.5-3.0 \mathrm{GHz}$. The frequency measurement range is given by the used measurement probe, and it covers the frequency range $0.7-1.3 \mathrm{GHz}$, which is currently considered the most suitable for MWI applications [22]. The relative permittivity and electrical conductivity of each sample were measured 10 times, and the measured data were supplemented with extended uncertainties with a coverage factor of $k=2$. Based on the measured dielectric properties of the samples, the composition of the mixtures was determined so that the dielectric properties correspond to the five tissue layers at $1 \mathrm{GHz}$ $[6,23]$, which is the center frequency of the MWI frequency range. The dielectric properties of each layer were determined from the IT'IS material parameter database V3.0 [24] as follows: scalp (weighted average of skin and fat with the ratio $2: 1$ ), skull (cortical skull), CSF (CSF), brain (average of gray matter, white matter, and cerebellum), hemorrhagic stroke (blood), and ischemic stroke (determined from the brain with $20 \%$ reduction of dielectric properties [6]). The tissues in parentheses are the ones listed in the IT'IS database.

2.3. Fabrication Process of the Head Phantom. The physical molds were printed on a 3D printer (Prusa i3 MK2, Prusa Research, Czech Republic) with $0.35 \mathrm{~mm}$ resolution in the longitudinal $(Z)$ direction using polyethylene terephthalate glycol (PETG) as the base material. All molds are depicted in Figure 3. Due to the elasticity of the molds, it was necessary to reinforce them with plaster. The surface of the molds was treated with the Polyurethane separators Ease Release 200 (Smooth-On, US), detergent Jar (Procter \& Gamble, US), or glossy adhesive tape to reduce the adhesion of the phantom mixtures. 


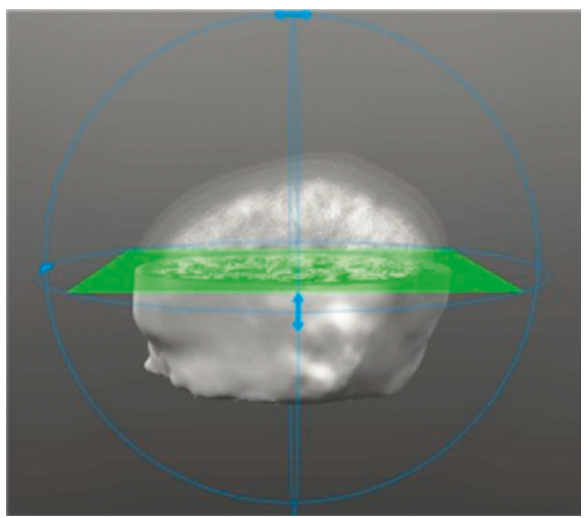

(a)

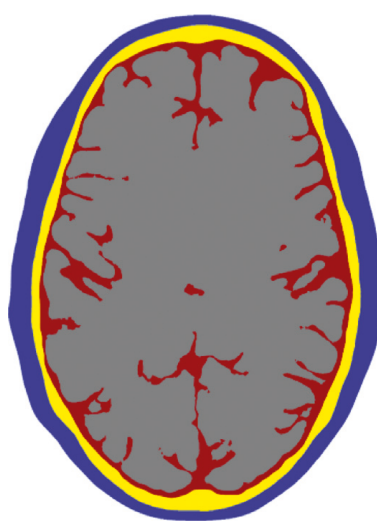

(b)

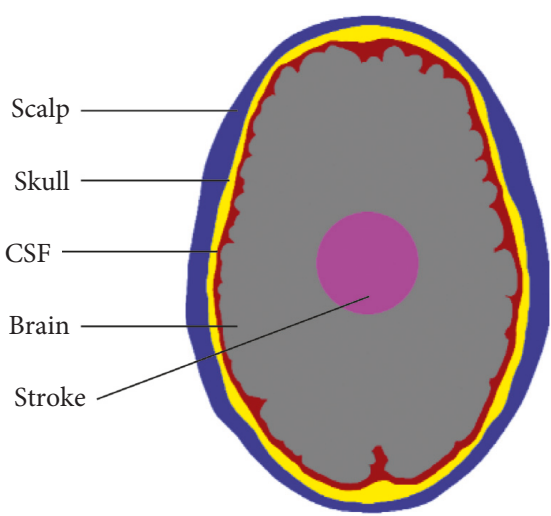

(c)

FIGURE 1: Baseline imaging for human head mold production: (a) cross section of the human head obtained from an MR image, (b) the 2D raw segmentation model of the main human head tissue layers, and (c) a simplified anatomical model with an added stroke model.

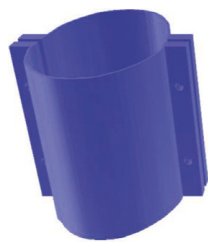

(a)

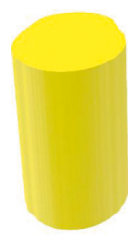

(b)

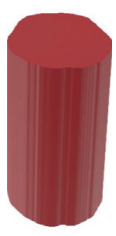

(c)

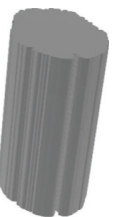

(d)

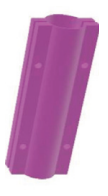

(e)

Figure 2: Computational design of the 2.5D human head tissue molds: scalp (a), skull (b), CSF (c), brain (d), and stroke (e) mold regions.

Table 1: Phantom materials to be used in the fabrication of the solid tissue layers of the human head phantom.

\begin{tabular}{lcc}
\hline Material & Model & Manufacturer \\
\hline Polyurethane rubber & PMC ${ }^{\circledR}-12130 /$ wet & Smooth-On, Inc. (US) \\
Graphite powder & 282863 -graphite & Sigma-Aldrich (US) \\
Carbon black powder & 45527 carbon black & Alfa Aesar (US) \\
Acetone & $99.9 \%$ acetone & Lach-Ner, s.r.o. (CZ) \\
\hline
\end{tabular}

TABLE 2: Phantom materials and their weight percentage used to prepare a series of samples to determine the most suitable composite to mimic the individual solid phantom layers of the human head physical model. For simplicity, the polyurethane rubber (\% by weight) is omitted.

\begin{tabular}{lccc}
\hline Series & Graphite powder (\% by weight) & Carbon black powder (\% by weight) & Acetone (\% by weight) \\
\hline 1 & $10,15,20,25,30,35,40,35,50$ & 0 & 0 \\
2 & 0 & $1,3,5,10,15$ & 0 \\
3 & 25 & $2,4,6$ & 0 \\
4 & $10,15,20,25,30$ & $2,4,6$ & 0 \\
5 & 20 & 0 & $10,20,30$ \\
6 & 40 & & 0 \\
\hline
\end{tabular}

The phantom mixtures were blended using a kitchen mixer (Bosch MUM 57860, Robert Bosch GmbH, Germany) with a dough kneading head for a total of 3 minutes to obtain a homogeneous phantom. The mixture was poured into the outer 3D printed mold (Figure 3(a)). Naturally occurring air bubbles were removed using a vacuum system. This is critical for dielectrically accurate phantoms since the presence of air would change the dielectric properties. The vacuum system consisted of a cylindrical vacuum chamber and an electric motor with a vacuum pump, where phantom mixtures were subjected to a vacuum of 0.8 bar three times for 30 seconds.

The inner 3D printed mold (Figure 3(b)) was slowly pressed into the homogeneous mixture to create the first layer (scalp). The insertion of inner molds had to be conducted with caution, and the position of the mold had to be controlled to guarantee that all layers were centered. The mixture was curated for 24 hours, and the internal mold was then carefully removed to avoid breaking the recently curated phantom layer. The same procedure was repeated with 


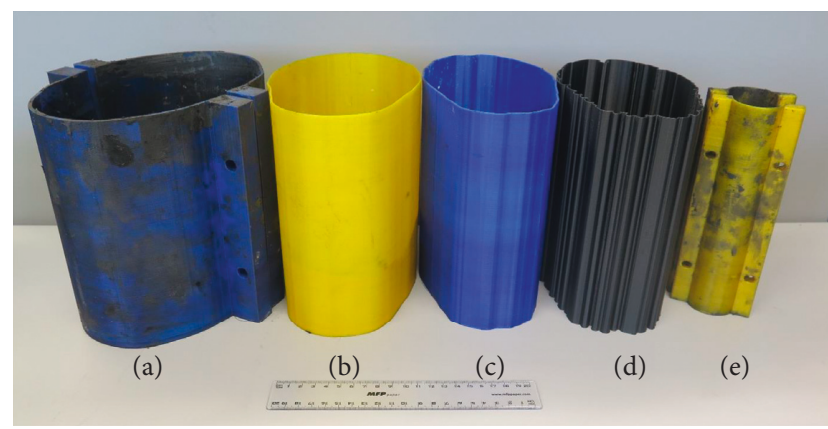

FIgURE 3: Molds printed in 3D for human head phantom fabrication: scalp (a), skull (b), CSF (c), gray matter (d), and stroke model (e).

the other two inner molds (Figures 3(c) and 3(d)) to create the skull and CSF domains. The resulting 3-layer solid phantom consisted of a waterproof container with an internal shape that mirrored the brain external anatomy. The container was then filled with a liquid brain phantom formed by adding deionized water and salt to isopropanol to achieve the desired brain dielectric properties [22]. Finally, the stroke phantom models were created by filling the cylindrical printed mold (Figure 3(e)) to mirror either ischemic or hemorrhagic tissue properties, yielding a total of 5 tissue layers.

\section{Results}

3.1. Phantom Materials and Dielectric Properties. The phantom mixtures that best represent the equivalent human tissue layers in terms of dielectric properties are summarized in Table 3. For simplicity, the dielectric properties are presented only at $1 \mathrm{GHz}$ in Table 4 for all tissue layers.

The frequency-dependent relative permittivity and electrical conductivity of the human head phantom layers are shown in Figures 4 and 5, respectively. The plots were split into different tissue layers for clarity.

3.2. Human Head Physical Phantom. Using 3D printed molds, we built anatomically and dielectrically realistic $2.5 \mathrm{D}$ phantoms of scalp, skull, and CSF, which resulted in a stable multilayer phantom depicted in Figure 6(a). The final 5-layer phantom is shown in Figure 6(b), which includes the liquid brain phantom and a stroke model with realistic electrical properties.

\section{Discussion}

The main goal of this work was to design and manufacture an anatomically and dielectrically realistic $2.5 \mathrm{D}$ 5-layer head phantom with the possibility of adding stroke phantoms with different electrical properties, shapes, sizes, and positions.

The materials used for the mold production were suitable to compact five tissue layers into a single phantom. The resulting mixture of Polyurethane rubber, graphite, and carbon black powder had sufficiently low viscosity for pouring into the mold and, after curing, provided a time-stable and partially flexible phantom. Furthermore, the phantom
TABLE 3: Weight percentages of phantom materials for fabrication of a multilayer human head phantom for stroke detection.

\begin{tabular}{lcccc}
\hline $\begin{array}{l}\text { Tissue } \\
\text { phantom }\end{array}$ & $\begin{array}{c}\text { Urethane } \\
\text { rubber (\%) }\end{array}$ & $\begin{array}{c}\text { Graphite } \\
\text { powder } \\
(\%)\end{array}$ & $\begin{array}{c}\text { Carbon black } \\
\text { powder (\%) }\end{array}$ & $\begin{array}{c}\text { Acetone } \\
(\%)\end{array}$ \\
\hline Scalp & 66.0 & 22.7 & 2.3 & 9.0 \\
Skull & 81.0 & 15.0 & 4.0 & 0 \\
CSF & 44.0 & 36.0 & 0 & 20.0 \\
$\begin{array}{l}\text { Hemorrhagic } \\
\text { stroke }\end{array}$ & 61.7 & 21.8 & 3.5 & 13.0 \\
$\begin{array}{l}\text { Ischemic } \\
\text { stroke }\end{array}$ & 53.6 & 37.3 & 0 & 9.1 \\
$\begin{array}{l}\text { Liquid brain } \\
\text { 61.15\% deionized water }+38 \% \\
\text { isopropanol + 0.85\% NaCl }\end{array}$ \\
\hline
\end{tabular}

TABle 4: Dielectric properties at $1 \mathrm{GHz}$ of the different tissueequivalent layers of the human head phantom for stroke detection. The measured data are supplemented with extended uncertainties $(k=2)$.

\begin{tabular}{lcccc}
\hline Tissue layer & $\begin{array}{c}\varepsilon_{r}(-) \\
\text { human }\end{array}$ & $\begin{array}{c}\mathcal{E}_{r}(-) \\
\text { measured }\end{array}$ & $\begin{array}{c}\sigma(\mathrm{S} / \mathrm{m}) \\
\text { human }\end{array}$ & $\begin{array}{c}\sigma(\mathrm{S} / \mathrm{m}) \\
\text { measured }\end{array}$ \\
\hline Scalp & 31.06 & $34.16 \pm 1.33$ & 0.64 & $0.77 \pm 0.08$ \\
Skull & 11.97 & $12.17 \pm 0.53$ & 0.09 & $0.11 \pm 0.01$ \\
CSF & 68.44 & $82.81 \pm 2.46$ & 2.46 & $2.99 \pm 0.04$ \\
Brain & 46.57 & $45.33 \pm 0.78$ & 0.97 & $0.98 \pm 0.01$ \\
$\begin{array}{l}\text { Ischemic } \\
\text { stroke }\end{array}$ & 37.26 & $36.43 \pm 0.66$ & 0.78 & $0.87 \pm 0.01$ \\
$\begin{array}{l}\text { Hemorrhagic } \\
\text { stroke }\end{array}$ & 61.08 & $55.78 \pm 2.18$ & 1.59 & $1.80 \pm 0.09$ \\
\hline
\end{tabular}

mixtures analyzed proved to be adequate to match the phantom with the human-equivalent dielectric properties over a wide range of frequencies relevant to microwave imaging.

The 3D printing technology proved to be suitable for fast manufacturing of phantom molds. Using PETG as the base material provided solid yet partially flexible molds. The mixtures stuck to the mold surface, and it was necessary to use a separator. The polyurethane separator Ease Release 200 did not work well with the PETG molds. The most suitable separator was the detergent Jar that was applied by brushing the entire mold surface or glossy adhesive tape which was suitable for flat surfaces. Nonetheless, even with the use of a separator, it was challenging to remove the mold after the mixture cured. At the same time, it was critical to remove unwanted air bubbles from the mixture by means of a vacuum chamber. Emerging bubbles could be observed on the surface of the samples, indicating an effective air extraction.

Phantom mixtures with higher concentrations of graphite powder presented higher viscosity, an effect that was even more pronounced with higher carbon black powder concentrations. The high viscosity formed lumps that made the mixture heterogeneous. To lower the viscosity, we added a small amount of acetone. However, acetone increased both dielectric properties making the dielectric properties matching between phantom and human tissues challenging. Nonetheless, the largest difference between phantom and human dielectric properties is lower 


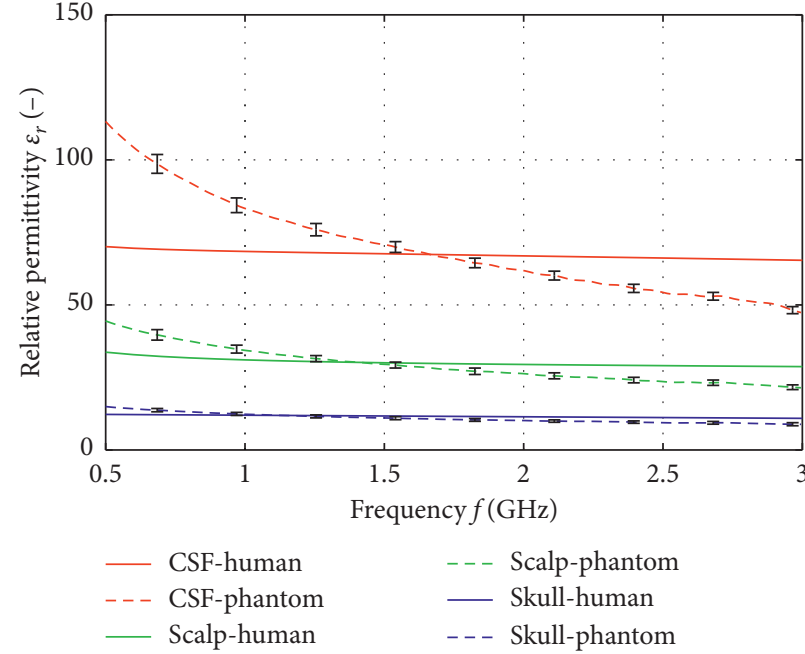

(a)

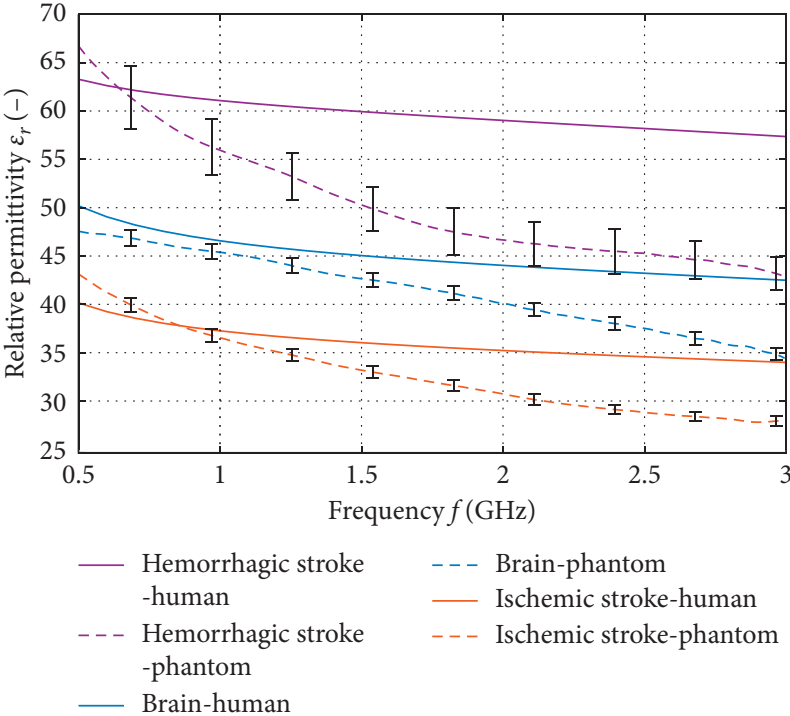

(b)

FIGURE 4: Frequency dependence of the human head phantom relative permittivity with extended uncertainties $(k=2)$ : (a) scalp, skull, and CSF solid phantom layers; (b) liquid brain and solid stroke phantom layers.

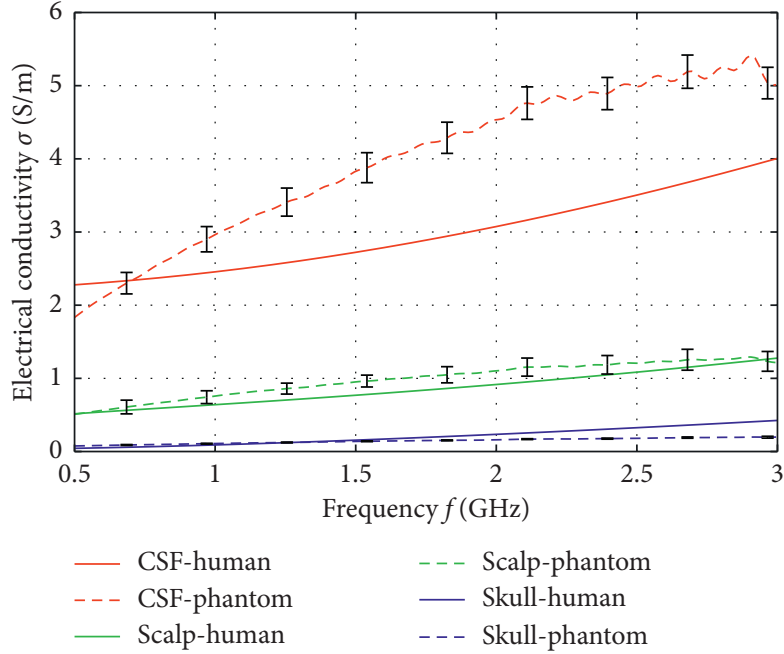

(a)

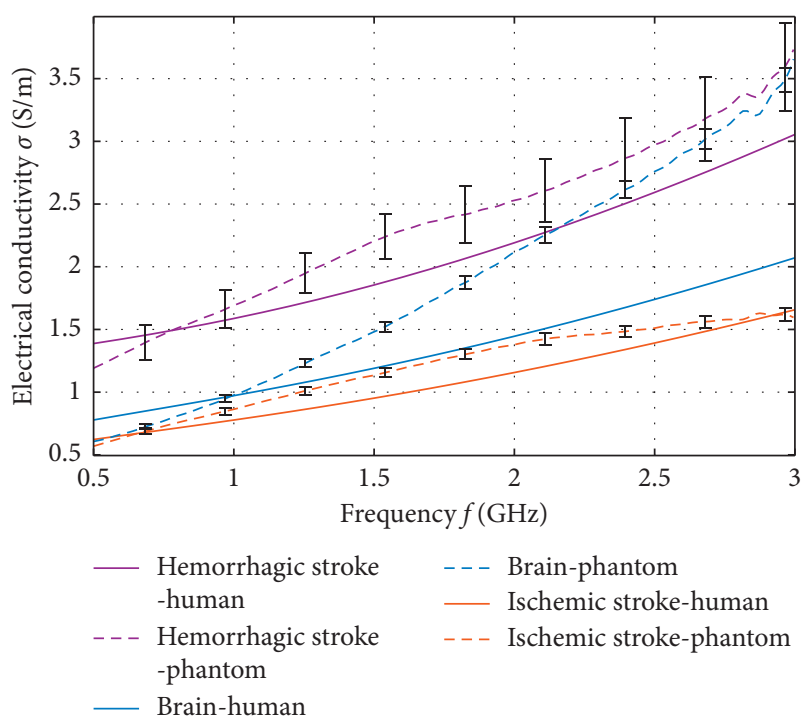

(b)

FIGURE 5: Frequency dependence of the human head phantom electrical conductivity with extended uncertainties $(k=2)$ : (a) scalp, skull and CSF solid phantom layers; (b) liquid brain and solid stroke phantom layers.

than $25 \%$ at $1 \mathrm{GHz}$ (Figures 4 and 5). Since the effect of relative permittivity on wave propagation is proportional to its square route, we consider the $25 \%$ difference acceptable. Moreover, the desired contrast in the dielectric properties between the different tissues in the human head phantom was preserved.

The dielectric properties of the solid phantoms were measured again one month after manufacturing, and they showed constant values. However, the liquid brain phantom changed its dielectric properties over time due to the evaporation of water and alcohol. Thus, we recommend measuring the dielectric properties of the mixture daily and, if necessary, adjust the composition or replace the mixture.

In reference [25], it was mentioned that extending the imaging system and the phantom of the head from $2.5 \mathrm{D}$ to $3 \mathrm{D}$ reduces the impact of the stroke area on the scattered EM field due to the significant reduction in stroke volume. On the other hand, the impact of the $2.5 \mathrm{D}$ phantom off the plane of the antenna array on the scattered field is not captured by the receiving antennas. Therefore, we believe that the $2.5 \mathrm{D}$ phantom [26] is a suitable platform for developing and testing algorithms, as well as microwave imaging hardware. 


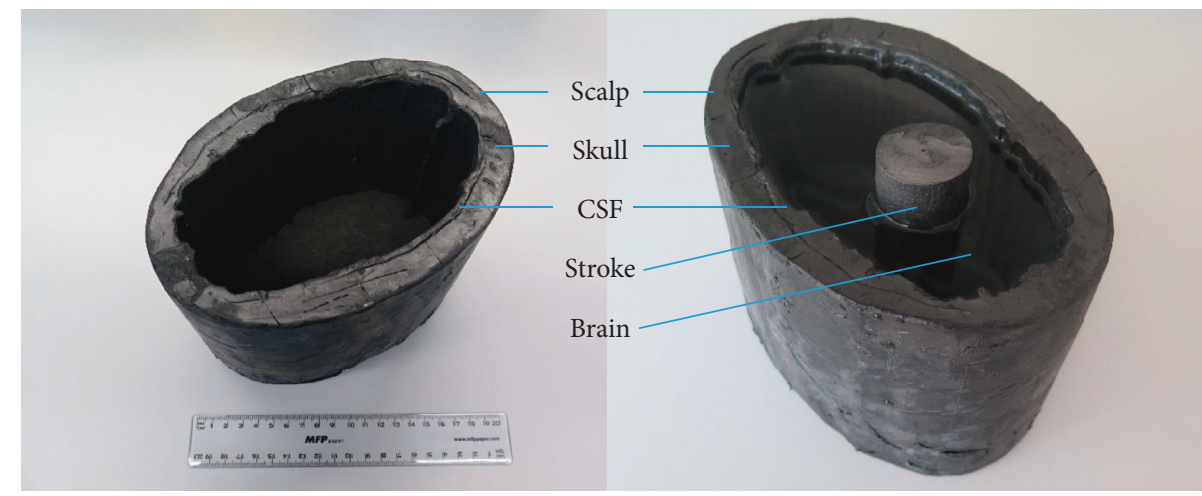

(a)

(b)

FIGURE 6: Photograph of the anatomically and dielectrically realistic 2.5D human head phantom: (a) external solid layers corresponding to scalp, skull, and CSF that are snugly attached to each forming a waterproof container; (b) fully assembled 5-layer human head phantom with added liquid brain phantom and a cylindrical solid stroke model.

However, the phantom production methodology described here can be used in the future to produce anatomically and dielectrically realistic $3 \mathrm{D}$ phantoms of the head.

\section{Conclusions}

An anatomically and dielectrically realistic phantom of the human head was designed and manufactured for microwave detection and classification of strokes. The phantom contains 5 different layers including the scalp, skull, CSF, brain regions, and a reconfigurable stroke model. The outermost three layers and the internal stroke model are made with solid mixtures that can be as thin as $1 \mathrm{~mm}$. The brain phantom was made in the liquid form to allow the insertion of different stroke models (ischemic or hemorrhagic) with different positions and shapes. The resulting structure of this head phantom resulted in a continuous multilayer environment without plastic walls between the different layers, making the phantom more dielectrically realistic. Furthermore, the phantom is time-stable and thus suitable for obtaining large measured data sets for testing new algorithms for stroke detection and classification based on machine learning methods.

\section{Data Availability}

The measured data used to support the findings of this study are available from the corresponding author upon request.

\section{Conflicts of Interest}

The authors declare that they have no conflicts of interest.

\section{Acknowledgments}

This work has been supported by a grant from the Czech Science Foundation (number 17-00477Y).

\section{References}

[1] National Heart, Lung, and Blood Institute, Stroke, National Heart, Lung, and Blood Institute, Bethesda, MD, USA, 2018, https://www.nhlbi.nih.gov/health-topics/stroke.
[2] M. A. Khorshidi, T. McKelvey, M. Persson, and H. D. Trefna, "Classification of microwave scattering data based on a subspace distance with application to detection of bleeding stroke," in Proceedings of the 2009 3rd IEEE International Workshop on Computational Advances in Multi-Sensor Adaptive Processing (CAMSAP), pp. 301-304, Dutch Antilles, The Netherlands, December 2009.

[3] Y. Wu, M. Zhu, D. Li, Y. Zhang, and Y. Wang, "Brain stroke localization by using microwave-based signal classification," in Proceedings of the 2016 International Conference on Electromagnetics in Advanced Applications (ICEAA), pp. 828-831, Queensland, Australia, September 2016.

[4] M. Pastorino, Wiley Series in Microwave and Optical Engineering: Microwave Imaging, John Wiley \& Sons, Hoboken, NJ, USA, 2010.

[5] S. Semenov, J. Kellam, P. Althausen et al., "Microwave tomography for functional imaging of extremity soft tissues: feasibility assessment," Physics in Medicine and Biology, vol. 52, no. 18, pp. 5705-5719, 2007.

[6] S. Semenov, T. Huynh, T. Williams, B. Nicholson, and A. Vasilenko, "Dielectric properties of brain tissue at $1 \mathrm{GHz}$ in acute ischemic stroke: experimental study on swine," Bioelectromagnetics, vol. 38, no. 2, pp. 158-163, 2017.

[7] M. Akter, T. Hirai, Y. Hiai et al., "Detection of hemorrhagic hypointense foci in the brain on susceptibility-weighted imaging," Academic Radiology, vol. 14, no. 9, pp. 1011-1019, 2007.

[8] B. A. J. Mohammed and A. M. Abbosh, "Realistic head phantom to test microwave systems for brain imaging," Microwave and Optical Technology Letters, vol. 56, no. 4, pp. 979-982, 2014.

[9] R. Scapaticci, L. Di Donato, I. Catapano, and L. Crocco, "A feasibility study on microwave imaging for brain stroke monitoring," Progress in Electromagnetics Research B, vol. 40, pp. 305-324, 2012.

[10] B. J. Mohammed, A. M. Abbosh, S. Mustafa, and D. Ireland, "Microwave system for head imaging," IEEE Transactions on Instrumentation and Measurement, vol. 63, no. 1, pp. 117-123, 2014.

[11] A. T. Mobashsher, A. M. Abbosh, and Y. Wang, "Microwave system to detect traumatic brain injuries using compact unidirectional antenna and wideband transceiver with verification on realistic head phantom," IEEE Transactions on Microwave Theory and Techniques, vol. 62, no. 9, pp. 18261836, 2014. 
[12] D. B. Rodrigues, P. F. Maccarini, S. Salahi et al., "Design and optimization of an ultra wideband and compact microwave antenna for radiometric monitoring of brain temperature," IEEE Transactions on Biomedical Engineering, vol. 61, no. 7, pp. 2154-2160, 2014.

[13] J. Garrett and E. Fear, "Stable and flexible materials to mimic the dielectric properties of human soft tissues," IEEE Antennas and Wireless Propagation Letters, vol. 13, pp. 599-602, 2014.

[14] C. Gabriel, "Tissue equivalent material for hand phantoms," Physics in Medicine and Biology, vol. 52, no. 14, pp. 4205-4210, 2007.

[15] H. Tamura, Y. Ishikawa, T. Kobayashi, and T. Nojima, “A dry phantom material composed of ceramic and graphite powder," IEEE Transactions on Electromagnetic Compatibility, vol. 39, no. 2, pp. 132-137, 1997.

[16] T. Kobayashi, T. Nojima, K. Yamada, and S. Uebayashi, "Dry phantom composed of ceramics and its application to SAR estimation," IEEE Transactions on Microwave Theory and Techniques, vol. 41, no. 1, pp. 136-140, 1993.

[17] B. McDermott, E. Porter, A. Santorelli et al., "Anatomically and dielectrically realistic microwave head phantom with circulation and reconfigurable lesions," Progress in Electromagnetics Research B, vol. 78, pp. 47-60, 2017.

[18] J. F. Gillooly, J. H. Brown, G. B. West, V. M. Savage, and E. L. Charnov, "Effects of size and temperature on metabolic rate," Science, vol. 293, no. 5538, pp. 2248-2251, 2001.

[19] E. G. Lee, R. L. Hadimani, and D. C. Jiles, Population Head Model Repository V1.0, IT'IS Foundation, Switzerland, 2016.

[20] E. G. Lee, W. Duffy, R. L. Hadimani et al., "Investigational effect of brain-scalp distance on the efficacy of transcranial magnetic stimulation treatment in depression," IEEE Transactions on Magnetics, vol. 52, no. 7, pp. 1-4, 2016.

[21] J. Tesarik, T. Pokorny, and L. Holek, "Samples of dry head tissues phantoms for brain stroke classification," in World Congress on Medical Physics and Biomedical Engineering 2018. IFMBE Proceedings, L. Lhotska, L. Sukupova, I. Lacković, and G. Ibbott, Eds., vol. 68/3, pp. 775-778, Springer, Singapore, 2019.

[22] J. Tesarik, L. F. Diaz Rondon, and O. Fiser, "Prototype of simplified microwave imaging system for brain stroke follow up," in World Congress on Medical Physics and Biomedical Engineering 2018. IFMBE Proceedings, L. Lhotska, L. Sukupova, I. Lacković, and G. Ibbott, Eds., vol. 68/3, pp. 771-774, Springer, Singapore, 2019.

[23] M. Persson, A. Fhager, H. D. Trefna et al., "Microwave-based stroke diagnosis making global prehospital thrombolytic treatment possible," IEEE Transactions on Biomedical Engineering, vol. 61, no. 11, pp. 2806-2817, 2014.

[24] IT'IS Foundation, Tissue Properties Database V3.0, IT'IS Foundation, Switzerland, 2015.

[25] R. Scapaticci, J. Tobon, G. Bellizzi, F. Vipiana, and L. Crocco, "Design and numerical characterization of a low-complexity microwave device for brain stroke monitoring," IEEE Transactions on Antennas and Propagation, vol. 66, no. 12, pp. 7328-7338, 2018.

[26] I. Merunka, O. Fiser, D. Vrba, and V. Jan, "Microwave tomography system for methodical testing of brain stroke detection approaches," International Journal of Antennas and Propagation, In press. 


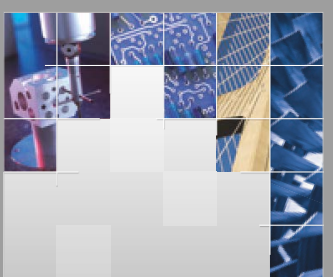

\section{Enfincering}
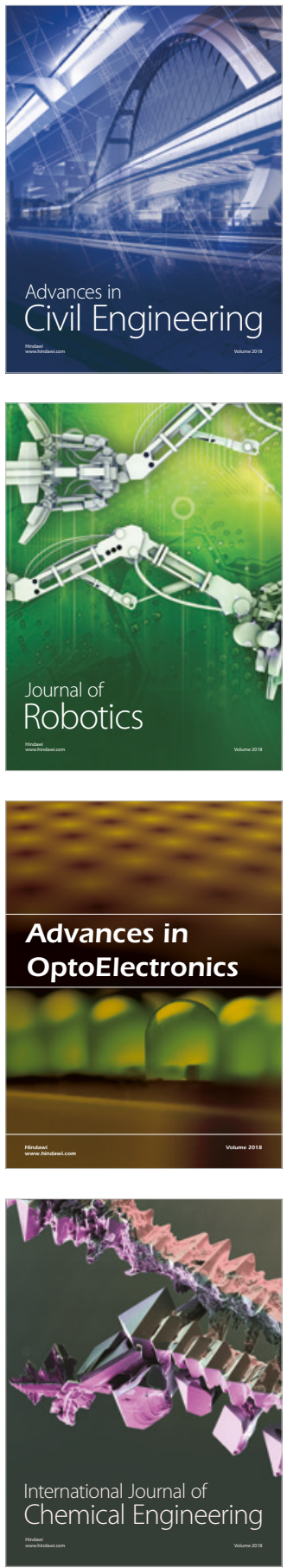

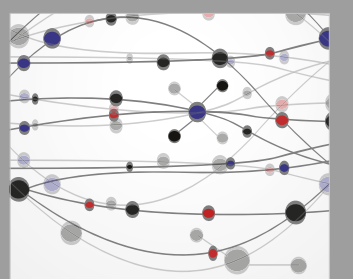

\section{Rotating \\ Machinery}

The Scientific World Journal

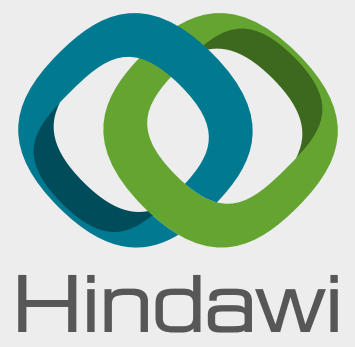

Submit your manuscripts at

www.hindawi.com
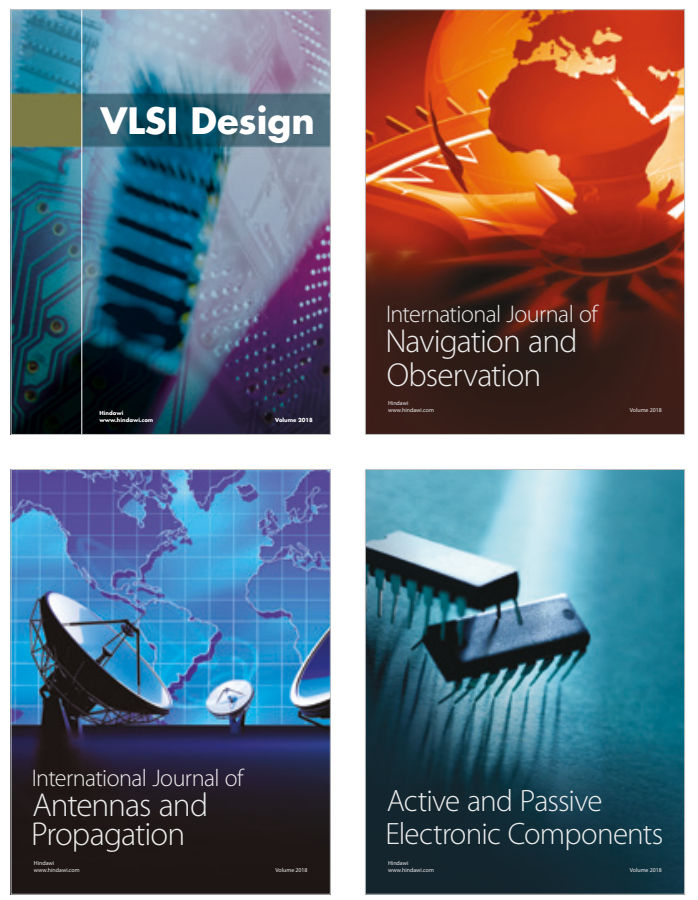
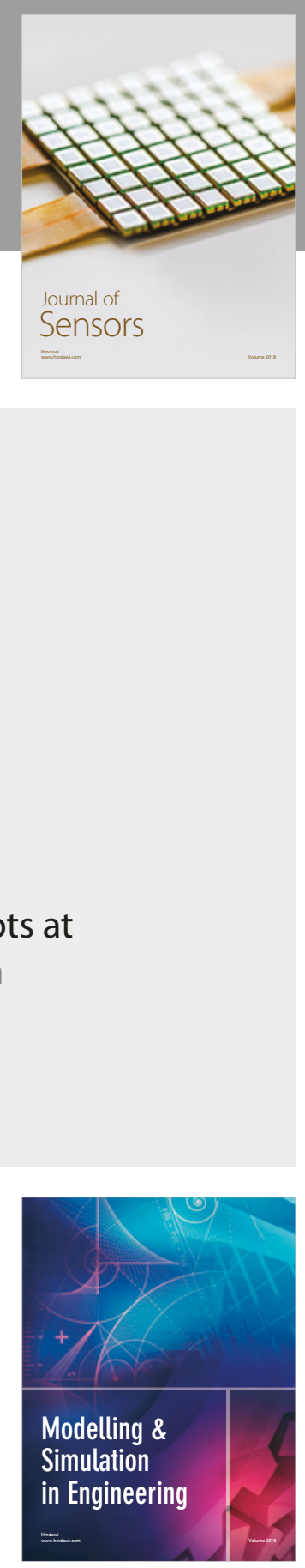

\section{Advances \\ Multimedia}
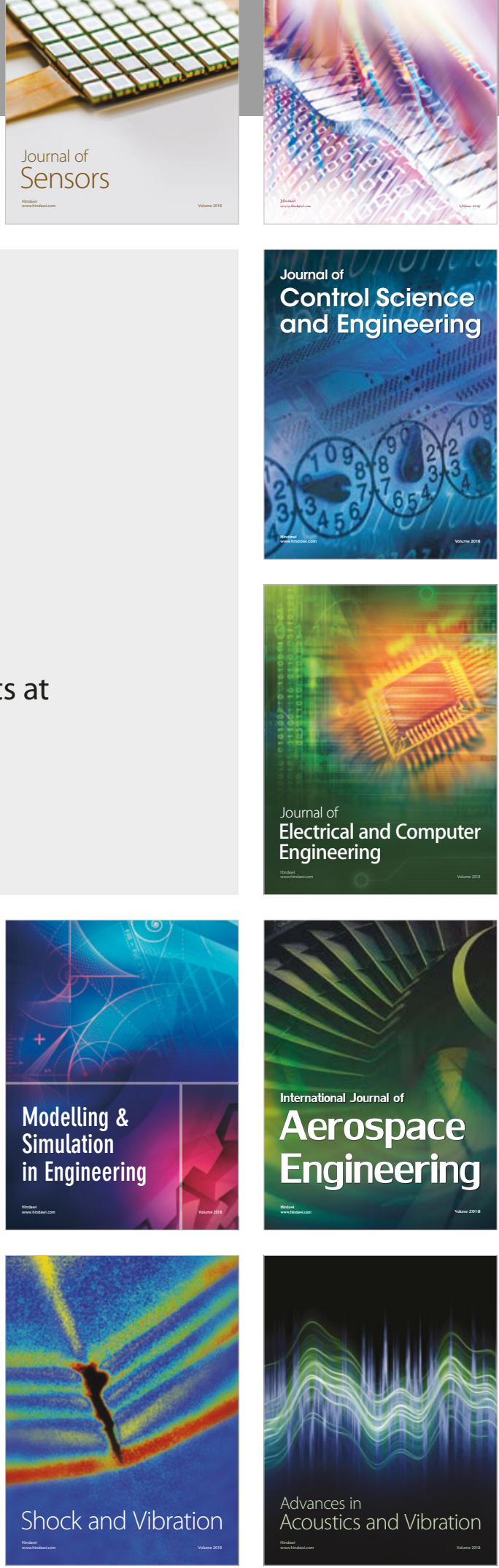